\title{
Task-Level Value Affects Trial-Level Reward Processing and Control
}

\author{
Cameron D. Hassall ${ }^{1}$ and Clay B. Holroyd $d^{2,3}$
}

${ }^{1}$ Department of Psychiatry, University of Oxford, Oxford, UK; ${ }^{2}$ Department of Experimental Psychology, Ghent University, Ghent, Belgium; ${ }^{3}$ Department of Psychology, University of Victoria, Victoria, BC; Correspondence: clay.holroyd@ugent.be

\begin{abstract}
Despite disagreement about how anterior cingulate cortex (ACC) supports decision making, a recent hypothesis suggests that activity in this region is best understood in the context of a task or series of tasks. One important task-level variable is average reward because it is both a known driver of effortful behaviour and an important determiner of the tasks in which we choose to engage. Here we asked how average task value affects reward-related ACC activity. To answer this question, we measured a reward-related signal said to be generated in ACC called the reward positivity (RewP) while participants gambled in three tasks of differing average value. The RewP was reduced in the high-value task, an effect that was not explainable by either reward magnitude or outcome expectancy. Average task value also affected cue-locked frontal midline theta (FMT), an ACC signal linked to cognitive control: FMT was enhanced for low-value cues relative to high-value cues. This effect was related to behaviour, as the more FMT a participant exhibited, the better they performed. These results suggest that ACC does not evaluate outcomes and cues in isolation, but in the context of the value of the current task.
\end{abstract}

Keywords: electroencephalography, reward positivity, anterior cingulate cortex, frontal midline theta 
The role of anterior cingulate cortex (ACC) in decision making has been hotly debated (Holroyd \& Verguts, 2021). According to one view, ACC supports reward-guided behaviours like foraging by encoding the value of switching from one task to another (Kolling et al., 2016). Others argue that ACC selects from among competing tasks by computing the expected value of control, balancing effort and task value (EVC: Shenhav et al., 2013). Computationally, ACC has been proposed to follow the principles of hierarchical reinforcement learning, exerting control over lower-level systems when ongoing rewards are worse than the average task value (Holroyd \& McClure, 2015).

In each of these approaches, ACC activity depends on task value. This claim has empirical support; neurons in monkey ACC, for example, encode both trial-by-trial reward and average task value (Amiez et al., 2006). But how does trial-level reward processing interact with task-level value processing in ACC? One possibility is via a reward prediction error (RPE), a concept borrowed from reinforcement learning ( $R L$ ) that indicates whether ongoing events are better or worse than expected (Sutton \& Barto, 2018). RPEs are proposed to be carried by the midbrain dopamine system to striatal and cortical targets (Schultz et al., 1997), including ACC (Amiez et al., 2005; Holroyd \& Coles, 2002). According to the standard "model-free" RL approach, called temporal difference learning, the RPE elicited by reward delivery is computed by subtracting the expected value of an action from the just-received reward. The standard reinforcement learning view is that RPEs are "one-step" computations, comparing the value of the current state to the value of the previous state only. Under this view, dopaminergic RPEs depend only on action/reward values, not the average task value.

Alternatively, midbrain dopamine activity may index a more general RPE rather than just an immediate, "one-step" RPE. Indeed, there is evidence that midbrain RPEs are sensitive to task context. For example, monkey dopamine neurons appear to track patterns in reward history beyond what would be predicted by a standard $R L$ algorithm (Nakahara et al., 2004). Reinforcement learning algorithms have therefore been augmented with internal models of the environment resulting in "model-based" RL algorithms. Both model-free and model-based reinforcement learning computations appear to drive activity of midbrain dopamine neurons (Collins \& Cockburn, 2020; Daw 
et al., 2011). There is therefore good reason to believe that midbrain RPE signals may be sensitive to task-level factors such as average task value.

In humans, midbrain RPE signals are thought to modulate ACC activity in a way that is measurable at the scalp. It has been proposed that a component of the event-related potential (ERP) called the reward positivity (RewP) varies as a function of RPE magnitude (Holroyd \& Coles, 2002; Sambrook \& Goslin, 2015; Walsh \& Anderson, 2014). In other words, the RewP provides a convenient readout of the degree to which outcomes differ from learned action values. Our goal here was to investigate whether and how reward-related ACC activity, as indexed by the RewP, varies with task value. Importantly, action value and task value are partially dissociable in reinforcement learning frameworks: High-value actions can occur in low-value tasks, and vice-versa.

To answer this question, we manipulated task value and action value by varying the proportion of "low-value" and "high-value" actions in three probabilistic learning tasks. EEG was recorded from participants as they attempted to learn correct actions for six predictive cues. Some of the cues were "high-value", indicating that a correct response would likely yield a reward. Other cues were "low-value", indicating that reward and nonreward outcomes were equally likely regardless of the response. We did not vary the value of the reward itself, which can affect the amplitude of the RewP (Kreussel et al., 2012; Sambrook \& Goslin, 2015). Rather, we varied the proportion of high- and lowvalue cues in each task: either all low-value, all high-value, or an even split. Our goal here was to vary reward at the task level while keeping trial-level rewards constant. Importantly, the same cue type (same reward expectancy and reward magnitude) appeared in multiple tasks, allowing us to isolate the effect of task value on feedbacklocked signals.

In addition to the RewP, we also examined the cue-locked ERP. There is evidence that reward-predicting cues can elicit a RewP-like signal (Holroyd et al., 2011; Krigolson et al., 2014). The computational explanation for this is that RPEs propagate backward in time to the earliest indicator that things are better or worse than expected. In a task with mixed high- and low-value cues, we might therefore expect high-value cues to elicit a positive prediction error (a positive RewP deflection) relative to low-value cues. 
Conversely, we would not expect a cue-locked prediction error to be elicited in a task with uniform cue values, because the cues all make the same prediction about upcoming rewards within the task. To summarize, trial cues ought to elicit positive/negative prediction errors in the "mixed value" task and no prediction error in the "uniform value" tasks

While investigating the cue-locked response, we observed what appeared to be differences in oscillatory activity. We then conducted an exploratory analysis examining frontal midline theta (FMT), which consists of oscillatory power in the 3-8 Hz range over frontal-central scalp locations. FMT (or FMO) is thought to originate in ACC and FMT is thought to index a cognitive control-related process (Cavanagh et al., 2012; Cavanagh \& Frank, 2014). A variety of events have been shown to elicit increased FMT power including loss feedback (Li et al., 2018) and predictive cues (Cavanagh et al., 2011). Like the RewP, FMT provides a readout of ACC activity, but one that is dissociable from the RewP (Hajihosseini \& Holroyd, 2013; Holroyd \& Umemoto, 2016; Paul et al., 2020). In particular, whereas the RewP reflects mainly phase-locked activity, FMT can reveal both phase-locked and non-phased-locked activity (Cohen et al., 2008).

\section{Method}

\section{Participants}

We tested 24 University of Victoria undergraduate participants with no known neurological impairments and with normal or corrected-to-normal vision (6 male, 4 lefthanded, 1 ambidextrous). Participant ages ranged from 18 to $26, M=20.7,95 \% \mathrm{Cl}$ $[19.8,21.6]$. Each participant received bonus credit in an undergraduate psychology course. Additionally, each participant received a performance-dependent monetary bonus. The study was approved by the University of Victoria Human Research Ethics Board, and all participants gave written informed consent.

This experiment required that participants learn to make optimal responses when this was possible, i.e., in response to high-value cues. As such, we applied the following a priori criterion: only the data of participants who made a correct response on at least $60 \%$ of the learnable trials in both the mid-value task and the high-value task were 
included in the main EEG analysis. 7 of the 24 participants did not meet this criterion and their data were therefore removed from the main analysis. However, the data of these 7 participants were included in a correlational analysis relating performance to FMT. Finally, the data of one participant were excluded from all analyses due to excessive EEG artifacts (across all conditions, the average trial rejection rate for this participant was $87 \%$ ). This left a total of 16 participants for the main analysis and 23 participants for the correlational analysis.

\section{Apparatus and Procedure}

Participants were seated $60 \mathrm{~cm}$ in front of an LCD display ( $60 \mathrm{~Hz}, 1024$ by 1280 pixels). Visual stimuli were presented using the Psychophysics Toolbox Extension (Brainard, 1997; Kleiner et al., 2007; Pelli, 1997) for MATLAB (Version 8.2, MathWorks, Natick, USA). Participants were given both verbal and written instructions in which they were asked to minimize head and eye movements.

Participants were told that they would be gambling in three different casinos. Each casino contained six different slot machines, and each slot machine was represented by a unique, coloured shape. Prior to "entering" a casino, the message "New Casino, New Coloured Shapes" was displayed. Shapes were reused across casinos, but with different colours (randomly chosen for each participant). The slot machines were described to participants as having two arms - a left arm and a right arm. Participants were instructed to, upon the appearance of a slot machine (a coloured shape), select one of the arms by pressing the corresponding key on a keyboard (the s-key to select the left arm, or the k-key to select the right arm). Participants were also told that their gamble would result in win or a loss - each outcome represented by a randomlyassigned fruit - and that for each slot machine "pulling one arm may be more likely to result in a win compared to pulling the other arm". Wins resulted in a gain of $\$ 0.03$, while losses resulted in no gain $(\$ 0.00)$. Participants were informed that their goal was to win as much money as possible and that they would be paid their total at the end of the experiment. 
Trials were grouped by casino, and each casino was entered only once. Casino order was counterbalanced with six possible casino orderings and four participants assigned to each ordering. Within a casino, participants encountered the 6 slot machines 24 times in random order (144 gambles). Unknown to participants, there were two types of slot machine: high-value and low-value. Each high-value slot machine had one arm (randomly chosen) that, when selected, would result in a win with $80 \%$ probability (selecting the other arm resulted in a win with a $20 \%$ probability). In contrast, low-value slot machines had no correct answer: there was a $50 \%$ probability of winning, regardless of which arm was pulled. The casinos contained either only low-value slot machines (the low-value task), high-value slot machines (the high-value task), or an even split between low- and high-value slot machines (the mid-value task). As each slot machine was encountered the same number of times, there was no learning advantage related to the number of exposures. Upon leaving a casino the total amount won within that casino was displayed.

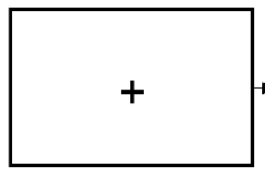

$400-600 \mathrm{~ms}$

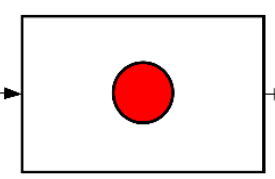

$1000 \mathrm{~ms}$

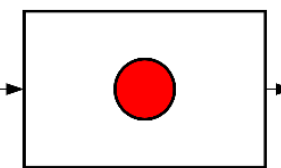

"beep" $50 \mathrm{~ms}$

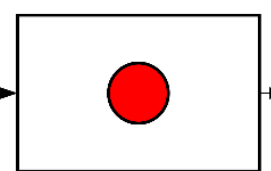

until response (up to $2000 \mathrm{~ms}$ )

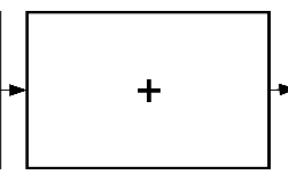

$400-600 \mathrm{~ms}$

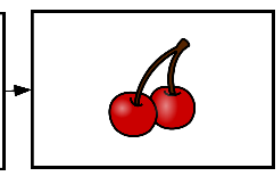

$1000 \mathrm{~ms}$

Figure 1. Task overview. After making a left or right button press, participants were shown fruit indicating the outcome (win or loss).

Each trial began with the appearance of a white fixation cross presented against a black background (Figure 1). The fixation cross, and all other visual stimuli, subtended $2^{\circ}$ of visual angle. After 400-600 ms, the fixation cross was replaced by the coloured shape representing the current trial's slot machine (the "cue"). After $1000 \mathrm{~ms}$, a $50 \mathrm{~ms} 400 \mathrm{~Hz}$ sine tone signalled participants to choose an arm (left or right) by pressing the corresponding key on a keyboard. The purpose of the delay between the cue and the tone was to isolate cue-related neural activity from response-related neural activity. The coloured shape/slot machine remained on the display until the participant responded (or until $2000 \mathrm{~ms}$ if no valid response was made). Finally, another fixation cross appeared 
for $400-600 \mathrm{~ms}$, followed by the feedback stimulus for $1000 \mathrm{~ms}$. Two images of fruit were used as feedback stimuli, chosen at random for each participant from six possible images. If the participant responded prior to the onset of the tone, the message "too fast" was displayed instead. If no response was made within the $2000 \mathrm{~ms}$ response window, or if a non-response key was pressed, the message "invalid" was displayed. In both cases (fast/invalid responses) the trial was excluded from both the behavioural analysis and the EEG analysis.

\section{Data Collection}

For each trial, the experimental software recorded reaction time (time since the onset of the tone), response (left, right, invalid, or no response), and casino/slot machine information. We also recorded casino value (low, mid, or high), slot machine value (low or high), trial outcome (win or lose) and - for high-value slot machines - whether or not the correct arm was chosen (that is, the arm more likely to result in a win).

EEG was recorded from $35 \mathrm{Ag} / \mathrm{AgCl}$ electrode locations using Brain Vision Recorder (Brain Products $\mathrm{GmbH}$, Munich, Germany). The electrodes were mounted in a fitted cap (EASYCAP GmbH, Herrsching, Germany) with a standard 10-20 layout and were recorded with respect to an average reference. Electrode impedances were lowered below $20 \mathrm{k} \Omega$ prior to recording and the EEG data were sampled at $250 \mathrm{~Hz}$ and amplified (Quick Amp, Brain Products GmbH, Munich, Germany).

\section{Data Analysis}

For all three tasks (low-value, mid-value, high-value) we computed the mean proportion of trials resulting in a win. For the mid-value and high-value tasks we computed, for each participant and task, the likelihood of a correct response to the high-value cues. (Recall that low-value cues had no correct response.) As mentioned previously, participants for whom this likelihood fell below $60 \%$, in either the mid-value task or the high-value task, were excluded from the main analyses (seven participants in total). For visualization purposes, we computed a sliding window measure of performance in the 
mid-value and high-value tasks: the proportion of wins in 30 trials, moving the window by 1 trial each time.

The EEG was analyzed in MATLAB 2020b (MathWorks, Natick, USA) using the EEGLAB library (Delorme \& Makeig, 2004). EEG data were first bandpass filtered (0.1$30 \mathrm{~Hz}, 60 \mathrm{~Hz}$ notch) and re-referenced to the average of the mastoid signals. Ocular artifacts were identified and removed using independent component analysis (ICA). The ICA was trained on three-second epoch starting at the presentation of the cue. Ocular components were identified using the iclabel function and removed from the continuous data. We removed components which iclabel determined were more likely to be eyerelated than brain-related.

\section{ERP Analysis}

We constructed 800 ms epochs around the appearance of each cue and feedback stimulus (-200 to $600 \mathrm{~ms}$ ). The data were then checked for remaining artifacts, and any epochs containing a change in voltage of more than $40 \mu \mathrm{V}$ per sample point or an overall change in voltage of more than $100 \mu \mathrm{V}$ across the epoch were excluded from the analysis. On average, $1.4 \%, 95 \% \mathrm{Cl}[0.5,2.2]$ of cue-locked epochs and $1.1 \%, 95 \% \mathrm{Cl}$ $[0.4,1.9]$ of feedback-locked epochs were excluded. We then averaged over the remaining epochs to create mean cue-locked, win-locked, and loss-locked waveforms for each task (low-value, mid-value, high-value), cue (low-value, high-value), and participant. To quantify the RewP, we used the difference wave method by subtracting the mean loss ERP from the corresponding mean win ERP for each task and cue. The difference wave approach is especially useful for isolating the RewP because it minimizes component overlap (Krigolson, 2017; Luck, 2014). We chose an electrode of interest using the "collapsed localizer" approach, averaging across all difference waves and locating the maximum scalp potential at FCz (Luck \& Gaspelin, 2017). We then identified a time window of interest by computing the times corresponding to $90 \%$ of the maximum scalp potential in the collapsed difference wave (240-276 ms post-feedback) ${ }^{1}$.

\footnotetext{
${ }^{1}$ This analysis window is somewhat earlier than the window prescribed by a RewP meta-analysis (240-340:

Sambrook \& Goslin, 2015). We chose our window to capture the apparent peak of the collapsed difference wave at
} 
The RewP was defined as the mean voltage recorded within this window at channel FCz for each task value, cue value, and participant. A similar approach was used for the cue-locked ERPs (see Supplemental Material).

\section{Time-Frequency Analysis}

To quantify FMT in response to cues, we first created cue-locked epochs from $600 \mathrm{~ms}$ pre-cue to 1200 ms post-cue. Compared to our ERP analysis, longer epochs were necessary to account for potential edge artifacts associated with time-frequency analysis. Using the same artifact rejection criteria as before, we excluded on average $3.33 \%, 95 \% \mathrm{Cl}[1.21,5.44]$ from analysis. We then transformed each cue-locked EEG epoch into its time-frequency representation. This was done by convolving the EEG with wavelets of the form $e^{i 2 \pi f t} e^{-t^{2} / 2 \sigma^{2}}$ for frequencies $f$ ranging from 1 to 30 in 60 logarithmic steps. The parameter $\sigma$, which affects wavelet width, was set to $6 / 2 \pi f$. The convolution was squared to compute power $p(t)$, averaged for each participant and condition, and converted to the decibel scale according to the equation $10 \log _{10}\left(\frac{p(t)}{p(\text { baseline })}\right)$. The baseline, $p$ (baseline), was defined as the mean power from 400 to -100 ms relative to the event of interest (cue or loss feedback). Grand averages were constructed for each condition by averaging time-frequency data across participants.

We focused on electrode $\mathrm{FC} z$, in line with previous work linking FMT with feedback processing and cognitive control (Cavanagh et al., 2012; Williams et al., 2021). As this was an exploratory analysis, we first collapsed the data over all cue conditions and then selected power values greater than 0 , for all times within $3-8 \mathrm{~Hz}$, corresponding to the frequency range used in previous studies (Cavanagh et al., 2012; Williams et al., 2021). We then computed the mean power within this cluster for each condition and participant. See Supplementary Figure 2 for the collapsed mean time-frequency response.

$258 \mathrm{~ms}$. Collapsed data was used to ensure that the resulting time window would not bias the results in favour of any of the conditions (Luck \& Gaspelin, 2017). 


\section{Inferential Statistics}

To confirm our task value manipulation, we compared the mean proportion of wins in each task using a one-way repeated-measures ANOVA. Partial and generalized etasquared were computed as:

$$
\eta_{p}{ }^{2}=S S_{\mathrm{v}} /\left(S S_{\mathrm{V}}+S S_{s \vee}\right) \quad \eta_{g}{ }^{2}=S S_{\mathrm{v}} /\left(S S_{\mathrm{V}}+S S_{S}+S S_{s} \mathrm{v}\right)
$$

where $S S_{V}$ is the sum of squares of the value effect (low, mid, high), $S S_{s v}$ is the error sum of squares of the value effect, and $S_{S}$ is the sum of squares between subjects. To test whether performance differed between the mid-value and high-value block - that is, whether participants learned about high-value cues differently - we used a repeatedmeasures $t$-test.

To determine the effect of average task value on ACC reward processing, we compared RewP scores using repeated measures $t$-tests. To avoid possible outcome frequency confounds, we only compared RewP scores from conditions with similar outcome frequencies (Krigolson, 2017). Specifically, we compared the low-task, low-cue RewP (infrequent rewards in an infrequent reward context) to the mid-task, low-cue RewP (infrequent rewards in a mid-frequency reward context). We then compared the midtask, high-cue RewP (frequent rewards in a mid-frequency reward context) to the hightask, high-cue RewP (frequent rewards in a frequent reward context).

To determine whether cues of different value elicited different adjustments to cognitive control, we compared FMT using repeated-measures t-tests. This was done for the task in which both cue types appeared (the mid-value task). We also compared cue-locked FMT to low-value cues in the low-value task to high-value cues in the high-value task. No other comparisons were made as this would require comparing events of differing frequency of occurrence, e.g., low-value cues were more frequent in the low-value task compared to the mid-value task. This is important because, like the RewP, FMT is sensitive to probability-related expectations (Cavanagh et al., 2012).

For all $t$-tests, we first checked the assumption of normality of each variable using the Shapiro-Wilk test. The assumption was met for all variables except for the RewP score 
in the high-task, high-cue condition. As the $t$-test is robust to non-normality, no correction was made. For each comparison, we also computed Cohen's $d$ for pairedsamples t-tests as:

$$
\text { Cohen's } d=\frac{M_{\text {diff }}}{s_{\text {diff }}}
$$

where $M_{\text {diff }}$ is the mean difference between the scores being compared and $s_{\text {diff }}$ is the standard deviation of the difference of the scores being compared (Cumming, 2014).

\section{Results}

\section{Behavioural Results}

The proportion of winning trials differed by task (low-value: $49.70 \%, 95 \% \mathrm{Cl}$ [47.73, 51.68], mid-value: $58.43 \%, 95 \% \mathrm{Cl}$ [56.51, 60.35], high-value: $68.84 \%, 95 \% \mathrm{Cl}$ [65.64, 72.24]), $F(2,30)=63.93, p<.001, \eta_{p}^{2}=0.81, \eta_{g}^{2}=0.72$. Note that since each win was worth the same amount, the total reward also differed between tasks (Figure 2a). There was no evidence of a difference between mean performance in the mid-value block $(73.67 \%, 95 \% \mathrm{Cl}[67.41,79.93])$ and mean performance in the high-value block (71.07\%, 95\% Cl [66.13, 78.02]), $t(15)=0.60, p=.557$, Cohen's $d=0.15$ (Figure $2 b-\mathrm{c}$ ). Recall that mean performance for both the mid-value blocks and the high-value blocks was defined as the proportion of high-value cues that were responded to correctly.

(a)

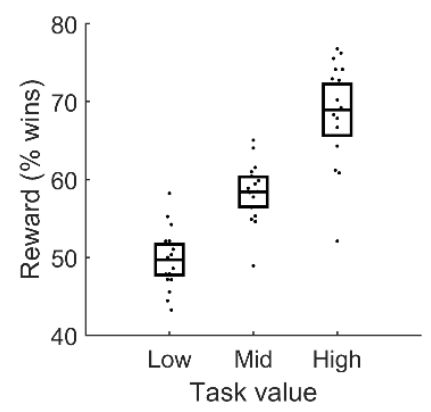

(b)

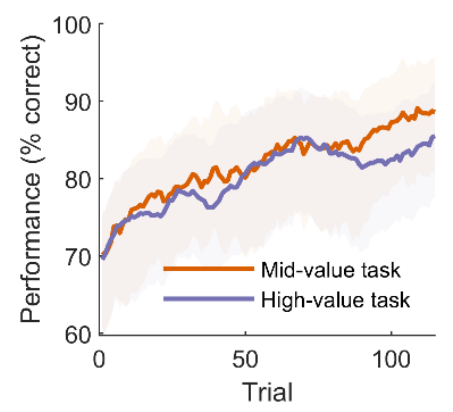

(c)

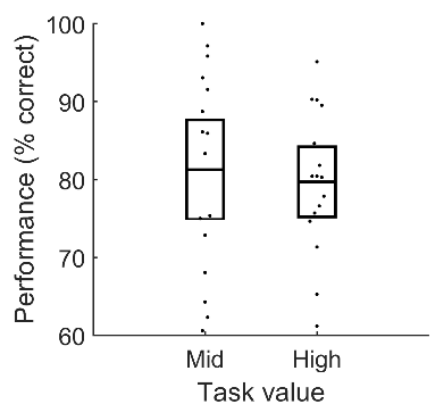

Figure 2. Behavioural results. (a) Mean proportion of wins in each task. (b) Performance curves in the tasks where correct responses were possible (mid-value and high-value tasks), indicating improvement 
over time. (c) Mean proportion of correct responses within the mid-value and high-value tasks. The shaded regions in each plot indicate $95 \%$ confidence intervals.

\section{Feedback-Locked EEG (RewP)}

After collapsing across the average feedback-locked waveforms (Figure 3a) we observed a scalp topography difference consistent with a RewP (Figure 3b). After constructing conditional difference waves (Figure 3c), we observed a significant effect of task value on the RewP amplitude elicited by high-value cues, indicating that RewP size was modulated by task value: The RewP following high-value cues was smaller in the high-value task $(5.09 \mu \mathrm{V}, 95 \% \mathrm{Cl}[3.15,7.04])$ than in the mid-value task $(7.63 \mu \mathrm{V}, 95 \%$ $\mathrm{Cl}[5.56,9.70]), t(15)=2.84, p=.012$, Cohen's $d=0.71$. The same difference was not observed when the RewP following low-value cues in the low-value task $(6.80,95 \% \mathrm{Cl}$ $[5.22,8.34])$ was compared to the RewP following low-value cues in the mid-value task $(6.61,95 \% \mathrm{Cl}[3.88,9.34], t(15)=0.16, p=.872$, Cohen's $d=0.04$ (Figure 3d).

(a)

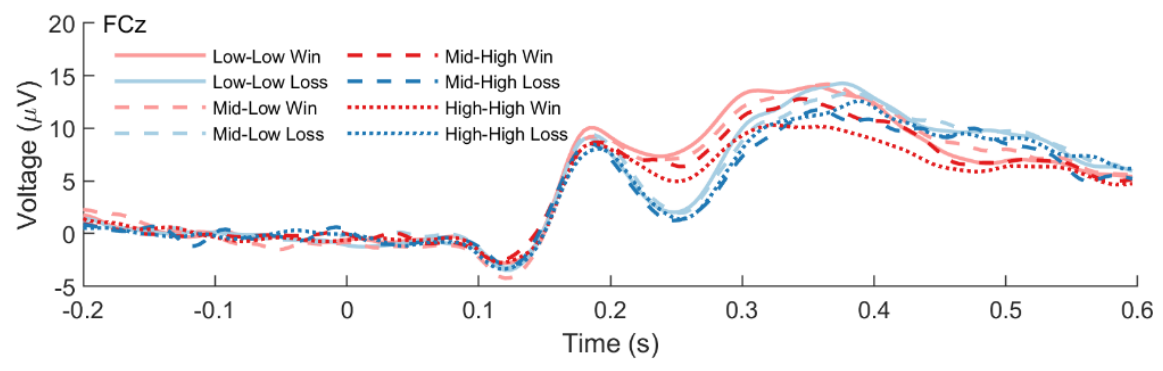

(c)

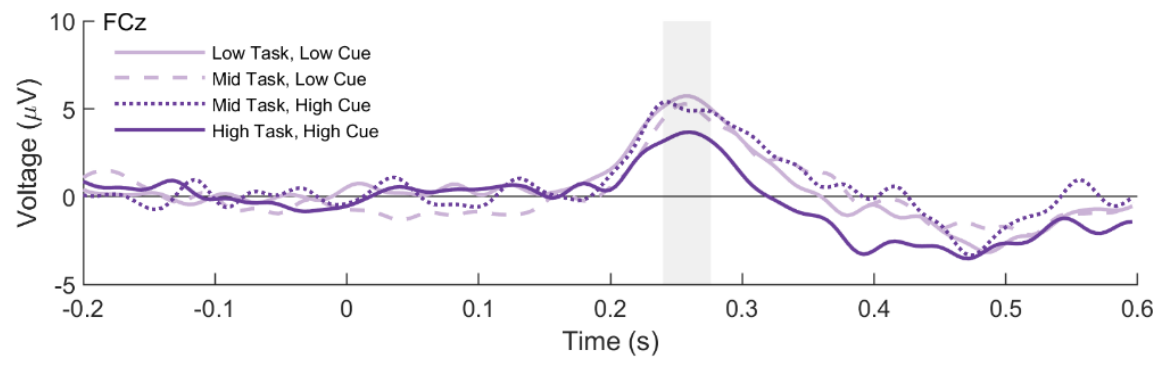

(b)

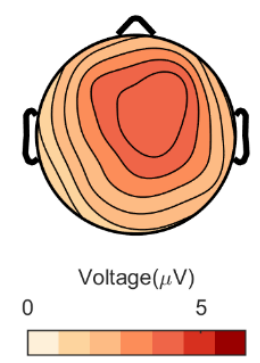

(d)

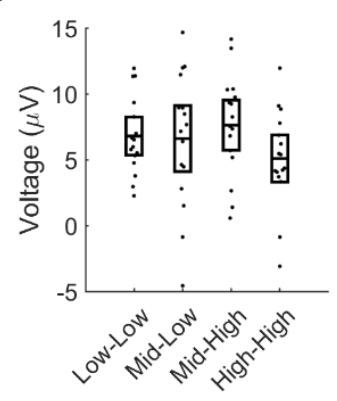

Figure 3. The reward positivity is reduced when task value is high. (a) Win/loss waveforms by task value (low, mid, high) and cue value (low, high). (b) Scalp distribution of the win-loss difference collapsed across conditions. (c) Difference waveforms by task value (low, mid, high) and cue value (low, high). The shaded area shows the region of analysis. (d) RewP scores by task value (low, mid, high) and cue value (low, high). Waveforms are time-locked to feedback onset. Error bars indicate 95\% confidence intervals. 


\section{Cue-Locked EEG (RewP and FMT)}

No cue-locked RewP was observed (see Supplemental Material for the cue-locked RewP results). However, clear FMT activity was observed in each condition (Figure 4a). Cue value affected FMT in the mid-value task (in which both low- and high-value cue types appeared): FMT to low-value cues $(0.38 \mathrm{~dB}, 95 \% \mathrm{Cl}[0.15,0.62])$ exceeded FMT to high-value cues $(0.03 \mathrm{~dB}, 95 \% \mathrm{Cl}[-0.30,0.35]), t(15)=2.45, p=0.027$, Cohen's $d=$ 0.61 . No statistically significant effect was observed between cue-locked FMT in the low-value $(0.43 \mathrm{~dB}, 95 \% \mathrm{Cl}[0.13,0.74])$ and high-value $(0.48,95 \% \mathrm{Cl}[-0.04,0.99])$ tasks, $t(15)=-0.29, p=.78$, Cohen's $d=-0.07$. (Figure $4 \mathrm{~b}$ ).

We then examined whether there was a relationship between FMT and performance. Because the goal of this analysis was to assess individual differences, the data of all of the participants (learners and non-learners) were included. In the mid-value task, we observed that performance correlated with FMT to low-value cues, $r(21)=0.48, p=.02$ but not high-value cues, $r(21)=0.08, p=.70$ (Figure $4 c-d$ ). In the high-value task, we observed that performance correlated with FMT to high-value cues, $r(21)=0.47, p=$ 0.02 (Figure 4e). 
(a)
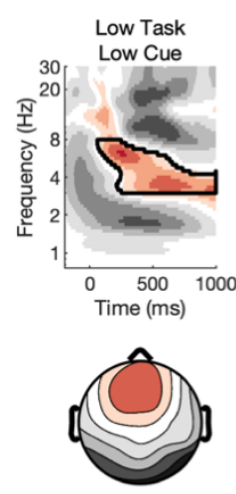

(c)
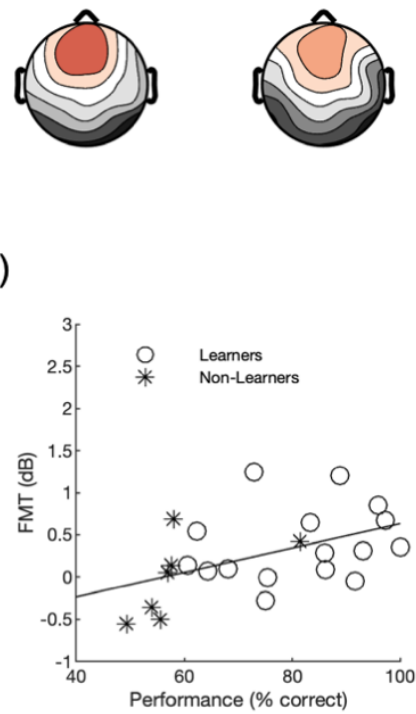

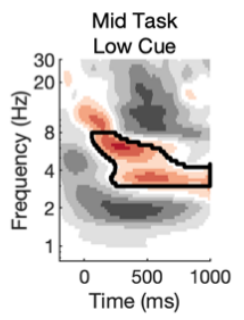

(d)
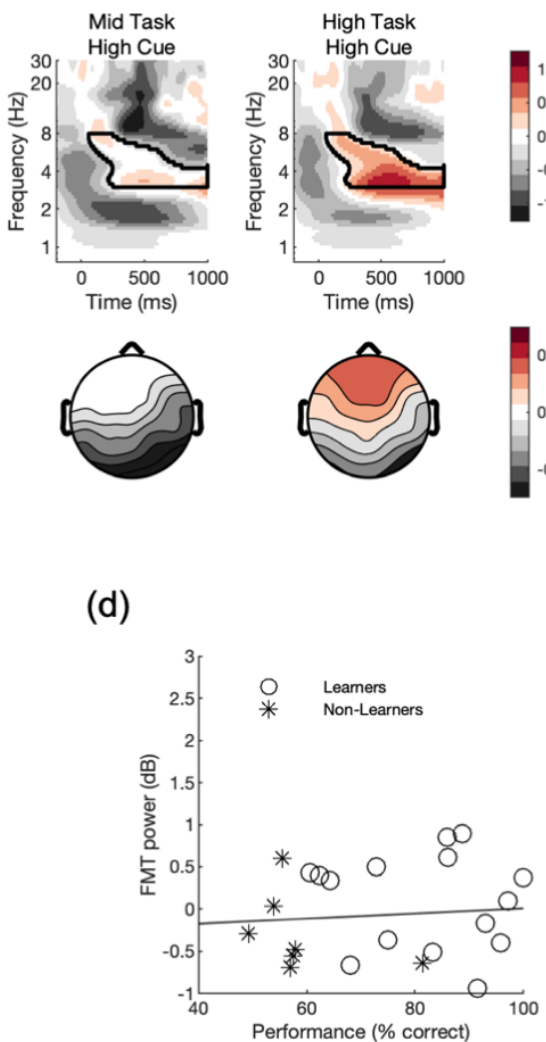

(b)
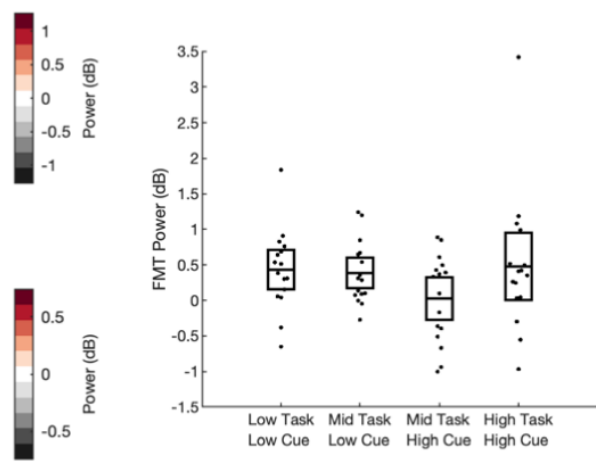

(e)

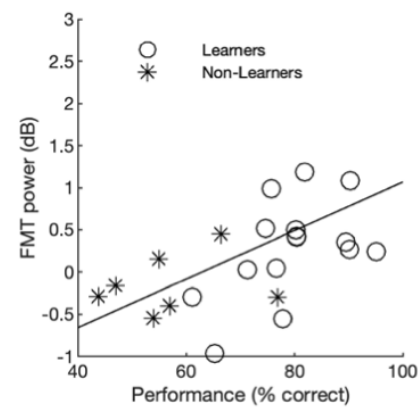

Figure 4. Cue-locked frontal midline theta (FMT) depends on cue and task value. (a) Time-frequency plots at FCz and scalp topographies for EEG locked to cues in each task (task value: low, mid, high; cue value: low, high). (b) Mean FMT for each task/cue with 95\% confidence intervals. Performance in the mid-value task correlated positively with low-value cue FMT (c) but not high-value cue FMT (d). (e) The relationship between cue-locked FMT and performance was also present in the high-value task.

\section{Discussion}

ACC activity is often studied by focussing on relatively short-term, within-trial processing of actions and events, despite empirical evidence and computational considerations suggesting that this region is more concerned about global aspects of task performance (Holroyd \& McClure, 2015; Holroyd \& Verguts, 2021; Holroyd \& Yeung, 2012; Umemoto et al., 2017). Here we show evidence that trial-to-trial ACC responses, as indexed by the RewP and FMT, are influenced by task value. We measured these signals in three tasks of varying average value. Despite matching outcomes for expectancy and triallevel value, the RewP was reduced when average task value was high. Task-level 
effects also propagated back to the cue: High- and low-value cues elicited differing levels of FMT, but only when they appeared together in the mid-value task.

These results are in line with a growing body of literature highlighting the importance of task context in understanding ACC activity. By "context" we mean task value (as in the present study) and other relevant task-level variables. For example, RewP amplitude is sensitive to the range of possible outcomes indicated by task instructions, not to the objective values of these outcomes (Holroyd et al., 2004). Task-level goals also matter: the amplitude of the RewP is greater when reward indicates progress towards a goal (Osinsky et al., 2017; Shahnazian et al., 2018). The RewP is also enhanced for selfrelevant rewards (Krigolson et al., 2013; Yu \& Zhou, 2006) and in agentic situations, when perceived control of outcomes is high (Hassall et al., 2019; Martin \& Potts, 2011; Mühlberger et al., 2017; Yeung et al., 2005).

As discussed, traditional RL models learn action values without representing task context (such as average task value) explicitly. However, here we controlled for triallevel expectancy and yet observed a task-level influence on the RewP. This result -a reduced RewP when task value is high - is inconsistent with a model that learns at the level of actions only, but may be consistent with an average-reward learning model in which prediction errors are defined as the difference between trial rewards and the average task value (e.g., Holroyd \& McClure, 2015). Under this view, the RewP ought to be reduced when trial outcomes are closer in value to the average task value, as we observed following high-value cues in the high-value task. Note that we did not observe a similar effect for low-value outcomes (outcomes following low-value cues). This result may be confounded by sense of agency, however, if our participants (in particular, "learners") became aware that low-value cues were unlearnable (Hassall et al., 2019; Martin \& Potts, 2011; Mühlberger et al., 2017; Sambrook \& Goslin, 2015; Yeung et al., 2005).

There is empirical evidence that the RewP is sensitive to average reward. For example, we have previously observed that a simple RL model fails to account for RewP amplitude when participants play virtual casino games in virtual casinos (Umemoto et 
al., 2017). There, a RewP was observed to casino game outcomes and to casinos themselves - in other words, ACC is sensitive to both game values and casino values. To explain these neural responses computationally, it was necessary to combine a simple RL model with an average-value learning model. In general, representing contextual information like average task value can be beneficial in a hierarchical framework in which a limited resource such as cognitive control needs to be regulated across multiple levels. These features are implemented in the hierarchical reinforcement learning (HRL) model of ACC (Holroyd \& McClure, 2015). Under this framework, ACC signals a need for control when outcomes are worse than the average task value.

Consistent with this possibility, we observed that FMT - a measure of ACC activity related to changes in cognitive control - was enhanced for low-value cues (corresponding to harder trials) relative to high-value cues (corresponding to easier trials) when both cues appeared in a mid-value task. Interestingly, this feature of ACC appears to be advantageous: FMT elicited by the low-value cues in the mid-level task was positively correlated with participant performance. Conversely, although high-value cues elicited a relative reduction in FMT in the mid-value task, we noted no relationship with performance in this case. This may be due to a floor effect on FMT, as suggested by the low FMT variability shown in Figure 4d. No floor effect was apparent in the highvalue task, however; here, FMT again predicted performance (Figure 4e).

The ACC has long been known to respond to trial-level events such as cues and rewards (Holroyd \& Coles, 2002). Another picture of the ACC is emerging - that of a region tasked with supporting extended sequences of actions (Holroyd \& Verguts, 2021; Holroyd \& Yeung, 2012). Here we provide evidence that ACC activity depends not only on trial-level features (is this a good outcome/cue?) but also task-level variables (how good is the task?) We suggest that a global view of ACC function will prove more fruitful than studying its neural responses at a molecular level. 


\section{Acknowledgements}

C.D.H. was supported by a Natural Sciences and Engineering Research Council of Canada (NSERC) Postdoctoral Fellowship (PDF-546078-2020). C.B.H. was supported by Natural Sciences and Engineering Research Council of Canada (NSERC) Grant \# 312409-05 and by funding from the European Research Council (ERC) under the EU's Horizon 2020 Research and Innovation Programme (grant agreement no. 787307). 


\section{References}

Amiez, C., Joseph, J. P., \& Procyk, E. (2005). Anterior cingulate error-related activity is modulated by predicted reward. European Journal of Neuroscience, 21(12), 3447-3452. https://doi.org/10.1111/j.1460-9568.2005.04170.x

Amiez, C., Joseph, J. P., \& Procyk, E. (2006). Reward Encoding in the Monkey Anterior Cingulate Cortex. Cerebral Cortex, 16(7), 1040-1055. https://doi.org/10.1093/cercor/bhj046

Brainard, D. H. (1997). The psychophysics toolbox. Spatial Vision, 10, 433-436.

Cavanagh, J. F., \& Frank, M. J. (2014). Frontal theta as a mechanism for cognitive control. Trends in Cognitive Sciences, 18(8), 414-421. https://doi.org/10.1016/j.tics.2014.04.012

Cavanagh, J. F., Wiecki, T. V., Cohen, M. X., Figueroa, C. M., Samanta, J., Sherman, S. J., \& Frank, M. J. (2011). Subthalamic nucleus stimulation reverses mediofrontal influence over decision threshold. Nature Neuroscience, 14(11), 1462-1467. https://doi.org/10/cx9fth

Cavanagh, J. F., Zambrano-Vazquez, L., \& Allen, J. J. B. (2012). Theta lingua franca: A common mid-frontal substrate for action monitoring processes. Psychophysiology, 49(2), 220-238. https://doi.org/10.1111/j.1469-8986.2011.01293.x

Cohen, M. X., Elger, C. E., \& Fell, J. (2008). Oscillatory Activity and Phase-Amplitude Coupling in the Human Medial Frontal Cortex during Decision Making. Journal of Cognitive Neuroscience, 21(2), 390-402. https://doi.org/10.1162/jocn.2008.21020

Collins, A. G. E., \& Cockburn, J. (2020). Beyond dichotomies in reinforcement learning. Nature Reviews Neuroscience, 21(10), 576-586. https://doi.org/10.1038/s41583-020-0355-6

Cumming, G. (2014). The New Statistics: Why and How. Psychological Science, 25(1), 7-29. https://doi.org/10.1177/0956797613504966

Daw, N. D., Gershman, S. J., Seymour, B., Dayan, P., \& Dolan, R. J. (2011). Model-Based Influences on Humans' Choices and Striatal Prediction Errors. Neuron, 69(6), 1204-1215. https://doi.org/10/fcnkgn

Delorme, A., \& Makeig, S. (2004). EEGLAB: An open source toolbox for analysis of single-trial EEG dynamics including independent component analysis. Journal of Neuroscience Methods, 134(1), 9-21. https://doi.org/10/bqr2f2

Hajihosseini, A., \& Holroyd, C. B. (2013). Frontal midline theta and N200 amplitude reflect complementary information about expectancy and outcome evaluation. Psychophysiology, 50(6), 550-562. https://doi.org/10/f4v4xk

Hassall, C. D., Hajcak, G., \& Krigolson, O. E. (2019). The importance of agency in human reward processing. Cognitive, Affective, \& Behavioral Neuroscience. https://doi.org/10/gf3tgc 
Holroyd, C. B., \& Coles, M. G. (2002). The neural basis of human error processing:

Reinforcement learning, dopamine, and the error-related negativity. Psychological Review, 109(4), 679. https://doi.org/10/bcr82b

Holroyd, C. B., Krigolson, O. E., \& Lee, S. (2011). Reward positivity elicited by predictive cues. Neuroreport, 22(5), 249-252. https://doi.org/10.1097/WNR.0b013e328345441d

Holroyd, C. B., Larsen, J. T., \& Cohen, J. D. (2004). Context dependence of the event-related brain potential associated with reward and punishment. Psychophysiology, 41(2), 245-253. https://doi.org/10.1111/j.1469-8986.2004.00152.x

Holroyd, C. B., \& McClure, S. M. (2015). Hierarchical control over effortful behavior by rodent medial frontal cortex: A computational model. Psychological Review, 122(1), 54-83. https://doi.org/10.1037/a0038339

Holroyd, C. B., \& Umemoto, A. (2016). The research domain criteria framework: The case for anterior cingulate cortex. Neuroscience \& Biobehavioral Reviews, 71, 418-443.

https://doi.org/10/f9gj32

Holroyd, C. B., \& Verguts, T. (2021). The Best Laid Plans: Computational Principles of Anterior Cingulate Cortex. Trends in Cognitive Sciences, 25(4), 316-329.

https://doi.org/10.1016/j.tics.2021.01.008

Holroyd, C. B., \& Yeung, N. (2012). Motivation of extended behaviors by anterior cingulate cortex. Trends in Cognitive Sciences, 16(2), 122-128. https://doi.org/10.1016/j.tics.2011.12.008

Kleiner, M., Brainard, D., Pelli, D., Ingling, A., Murray, R., \& Broussard, C. (2007). What's new in Psychtoolbox-3. Perception, 36(14), 1.

Kolling, N., Wittmann, M. K., Behrens, T. E. J., Boorman, E. D., Mars, R. B., \& Rushworth, M. F. S. (2016). Value, search, persistence and model updating in anterior cingulate cortex. Nature Neuroscience, 19(10), 1280-1285. https://doi.org/10/f856kc

Kreussel, L., Hewig, J., Kretschmer, N., Hecht, H., Coles, M. G. H., \& Miltner, W. H. R. (2012). The influence of the magnitude, probability, and valence of potential wins and losses on the amplitude of the feedback negativity. Psychophysiology, 49(2), 207-219.

https://doi.org/10.1111/j.1469-8986.2011.01291.x

Krigolson, O. E. (2017). Event-related brain potentials and the study of reward processing: Methodological considerations. International Journal of Psychophysiology. https://doi.org/10.1016/j.ijpsycho.2017.11.007

Krigolson, O. E., Hassall, C. D., Balcom, L., \& Turk, D. (2013). Perceived ownership impacts reward evaluation within medial-frontal cortex. Cognitive, Affective, \& Behavioral Neuroscience, 13(2), 262-269. https://doi.org/10.3758/s13415-012-0144-4 
Krigolson, O. E., Hassall, C. D., \& Handy, T. C. (2014). How We Learn to Make Decisions: Rapid Propagation of Reinforcement Learning Prediction Errors in Humans. Journal of Cognitive Neuroscience, 26(3), 635-644. https://doi.org/10/gd32z4

Li, P., Peng, W., Li, H., \& Holroyd, C. B. (2018). Electrophysiological measures reveal the role of anterior cingulate cortex in learning from unreliable feedback. Cognitive, Affective, \& Behavioral Neuroscience, 1-15. https://doi.org/10.3758/s13415-018-0615-3

Luck, S. J. (2014). An introduction to the event-related potential technique (Second edition). The MIT Press.

Luck, S. J., \& Gaspelin, N. (2017). How to get statistically significant effects in any ERP experiment (and why you shouldn't). Psychophysiology, 54(1), 146-157. https://doi.org/10.1111/psyp.12639

Martin, L. E., \& Potts, G. F. (2011). Medial Frontal Event Related Potentials and Reward Prediction: Do Responses Matter? Brain and Cognition, 77(1), 128-134.

https://doi.org/10/fcgftx

Mühlberger, C., Angus, D. J., Jonas, E., Harmon-Jones, C., \& Harmon-Jones, E. (2017). Perceived control increases the reward positivity and stimulus preceding negativity. Psychophysiology, 54(2), 310-322. https://doi.org/10.1111/psyp.12786

Nakahara, H., Itoh, H., Kawagoe, R., Takikawa, Y., \& Hikosaka, O. (2004). Dopamine Neurons Can Represent Context-Dependent Prediction Error. Neuron, 41(2), 269-280.

https://doi.org/10.1016/S0896-6273(03)00869-9

Osinsky, R., Ulrich, N., Mussel, P., Feser, L., Gunawardena, A., \& Hewig, J. (2017). The Feedbackrelated Negativity Reflects the Combination of Instantaneous and Long-term Values of Decision Outcomes. Journal of Cognitive Neuroscience, 29(3), 424-434. https://doi.org/10/gdk3pj

Paul, K., Vassena, E., Severo, M. C., \& Pourtois, G. (2020). Dissociable effects of reward magnitude on fronto-medial theta and FRN during performance monitoring. Psychophysiology, 57(2), e13481. https://doi.org/10.1111/psyp.13481

Pelli, D. G. (1997). The VideoToolbox software for visual psychophysics: Transforming numbers into movies. Spatial Vision, 10(4), 437-442. https://doi.org/10.1163/156856897X00366

Sambrook, T. D., \& Goslin, J. (2015). A neural reward prediction error revealed by a metaanalysis of ERPs using great grand averages. Psychological Bulletin, 141(1), 213-235. https://doi.org/10/f6whtb

Schultz, W., Dayan, P., \& Montague, P. R. (1997). A Neural Substrate of Prediction and Reward. Science, 275(5306), 1593-1599. https://doi.org/10.1126/science.275.5306.1593 
Shahnazian, D., Shulver, K., \& Holroyd, C. B. (2018). Electrophysiological responses of medial prefrontal cortex to feedback at different levels of hierarchy. Neurolmage, 183, 121-131. https://doi.org/10.1016/j.neuroimage.2018.07.064

Shenhav, A., Botvinick, M. M., \& Cohen, J. D. (2013). The Expected Value of Control: An Integrative Theory of Anterior Cingulate Cortex Function. Neuron, 79(2), 217-240. https://doi.org/10/f45wsh

Sutton, R. S., \& Barto, A. G. (2018). Reinforcement learning: An introduction (Second edition). The MIT Press.

Umemoto, A., HajiHosseini, A., Yates, M. E., \& Holroyd, C. B. (2017). Reward-based contextual learning supported by anterior cingulate cortex. Cognitive, Affective, \& Behavioral Neuroscience, 17(3), 642-651. https://doi.org/10.3758/s13415-017-0502-3

Walsh, M. M., \& Anderson, J. R. (2014). Navigating complex decision spaces: Problems and paradigms in sequential choice. Psychological Bulletin, 140(2), 466-486.

https://doi.org/10.1037/a0033455

Williams, C. C., Ferguson, T. D., Hassall, C. D., Abimbola, W., \& Krigolson, O. E. (2021). The ERP, frequency, and time-frequency correlates of feedback processing: Insights from a large sample study. Psychophysiology, 58(2), e13722. https://doi.org/10.1111/psyp.13722

Yeung, N., Holroyd, C. B., \& Cohen, J. D. (2005). ERP Correlates of Feedback and Reward Processing in the Presence and Absence of Response Choice. Cerebral Cortex, 15(5), 535-544. https://doi.org/10.1093/cercor/bhh153

Yu, R., \& Zhou, X. (2006). Brain responses to outcomes of one's own and other's performance in a gambling task. Neuroreport, 17(16), 1747-1751.

https://doi.org/10.1097/01.wnr.0000239960.98813.50 